Article

\title{
Planar Harmonic and Monogenic Polynomials of Type $A$
}

\section{Charles F. Dunkl}

Department of Mathematics, University of Virginia, P.O. Box 400137, Charlottesville, VA 22904-4137, USA; cfd5z@virginia.edu

Academic Editor: Hari M. Srivastava

Received: 8 September 2016; Accepted: 14 October 2016; Published: 21 October 2016

\begin{abstract}
Harmonic polynomials of type $A$ are polynomials annihilated by the Dunkl Laplacian associated to the symmetric group acting as a reflection group on $\mathbb{R}^{N}$. The Dunkl operators are denoted by $T_{j}$ for $1 \leq j \leq N$, and the Laplacian $\Delta_{\kappa}=\sum_{j=1}^{N} T_{j}^{2}$. This paper finds the homogeneous harmonic polynomials annihilated by all $T_{j}$ for $j>2$. The structure constants with respect to the Gaussian and sphere inner products are computed. These harmonic polynomials are used to produce monogenic polynomials, those annihilated by a Dirac-type operator.
\end{abstract}

Keywords: Dunkl operators; symmetric group; harmonic polynomials

\section{Introduction}

The symmetric group $\mathcal{S}_{N}$ acts on $x \in \mathbb{R}^{N}$ as a reflection group by permutation of coordinates. The group is generated by reflections in the mirrors $\left\{x: x_{i}=x_{j}, i<j\right\}$. The function $w_{\kappa}(x)=\prod_{1 \leq i<j \leq N}\left|x_{i}-x_{j}\right|^{2 \kappa}$ with parameter $\kappa$ is invariant under this action, and, for $\kappa>-\frac{1}{N}$, there are several measures that incorporate $w_{\mathcal{K}}$ and give rise to interesting orthogonality structures. The corresponding measure on the $N$-torus is related to the Calogero-Sutherland quantum-mechanical model of $N$ identical particles on the circle with $1 / r^{2}$ interaction potential, and the measure $w_{\mathcal{K}}(x) e^{-|x|^{2} / 2} \mathrm{~d} x$ is related to the model of $N$ identical particles on the line with $1 / r^{2}$ interactions and harmonic confinement (see [1] (Section 11.6)). This paper mainly concerns the measure on the unit sphere in $\mathbb{R}^{N}$ for which there is an orthogonal decomposition involving harmonic polynomials. In the present setting, harmonic refers to the Laplacian operator $\Delta_{\kappa}$ produced by the type- $A$ Dunkl operators.

For $x \in \mathbb{R}^{N}$ and $\{i, j\} \subset\{1,2, \ldots, N\}$ set $x(i, j)=\left(\ldots, x_{j}, \ldots, x_{i}, \ldots\right)$, that is, entries \#i and \#j are interchanged.

Definition 1. For a polynomial $f$ and $1 \leq i \leq N$

$$
\begin{aligned}
T_{i} f(x) & :=\frac{\partial}{\partial x_{i}} f(x)+\kappa \sum_{j=1, j \neq i}^{N} \frac{f(x)-f(x(i, j))}{x_{i}-x_{j}} \\
\Delta_{\kappa} f(x) & :=\sum_{i=1}^{N} T_{i}^{2} .
\end{aligned}
$$

The (Dunkl) operators $T_{i}$ mutually commute and map polynomials to polynomials. The background for the theory can be found in the treatise ([1] (Chapter 6, Chapter 10.2)). An orthogonal basis for $L^{2}\left(\mathbb{R}^{N}, w_{\kappa}(x) e^{-|x|^{2} / 2} \mathrm{~d} x\right)$ can be defined in terms of products $f(x) L_{n}^{\lambda}\left(|x|^{2} / 2\right)$ where $f$ comes from an orthogonal set of harmonic homogeneous polynomials and the Laguerre polynomial 
index $\lambda=\operatorname{deg} f-1+\frac{N}{2}((N-1) \kappa+1)$ (for the theory of Laguerre polynomials, see Szegö [2] (Section 5.1)). However, attempts to explicitly construct harmonic polynomials run into technical complications, presumably due to the fact that the sign-changes (example: $x \mapsto\left(-x_{1}, x_{2}, \ldots, x_{N}\right)$ ) are not elements of the symmetry group and thus the $+/-$ symmetry of $T_{i}^{2}$ can not be used. To start on the construction problem, we will determine all the harmonic homogeneous polynomials annihilated by $T_{j}$ for $2<j \leq N$. They are the analogues of ordinary harmonic polynomials in two variables and thus we call them planar. In this situation, there is a natural symmetry based on the transposition $(1,2)$ : polynomials $f$ satisfying $f(x(1,2))=f(x)$ are called symmetric and those satisfying $f(x(1,2))=-f(x)$ are called antisymmetric. Then, $T_{1}+T_{2}$ preserves the symmetry type and $T_{1}-T_{2}$ reverses it. This property is relevant since $T_{1}^{2}+T_{2}^{2}=\frac{1}{2}\left(T_{1}+T_{2}\right)^{2}+\frac{1}{2}\left(T_{1}-T_{2}\right)^{2}$.

Section 2 describes the basis of polynomials used in the construction, sets up and solves the recurrence equations required to produce symmetric and antisymmetric harmonic polynomials. In addition, the formulae for the actions of $T_{1} \pm T_{2}$ on the harmonics are derived. In Section 3, the inner product structures involving the weight function $w_{\kappa}$ are defined and the structural constants for the harmonic polynomials are computed. By means of Clifford algebra techniques, one can define an operator of Dirac type and Section 4 describes this theory and produces the planar monogenic polynomials. Finally, Section 5 contains technical material providing proofs for some of the results appearing in Sections 2 and 3.

\section{The $p$-Basis and Construction of Harmonic Polynomials}

The natural numbers $\{0,1,2,3 \ldots\}$ are denoted by $\mathbb{N}_{0}$. The largest integer $\leq t \in \mathbb{R}$ is denoted by $\lfloor t\rfloor$. Suppose $f$ is a polynomial in $x \in \mathbb{R}^{N}$ then $(1,2) f$ denotes the polynomial $f(x(1,2))$. To facilitate working with generating functions, we introduce the notation coef $\left(f, g_{j}\right):=c_{j}$ for the designated coefficient of $f$ in the expansion $f=\sum_{i} c_{i} g_{i}$ in terms of a basis $\left\{g_{i}\right\}$. Throughout the paper $\kappa$ is a fixed parameter, implicit in $\left\{T_{i}\right\}$, generally subject to $\kappa>-\frac{1}{N}$.

The $p$-basis associated with the operators $\left\{T_{i}\right\}$ is constructed as follows: for $1 \leq i \leq N$, the polynomials $p_{n}\left(x_{i} ; x\right)$ are given by the generating function

$$
\sum_{n=0}^{\infty} p_{n}\left(x_{i} ; x\right) r^{n}=\left(1-r x_{i}\right)^{-1} \prod_{j=1}^{N}\left(1-r x_{j}\right)^{-\kappa}
$$

then, for $\alpha=\left(\alpha_{1}, \ldots, \alpha_{N}\right) \in \mathbb{N}_{0}^{N}$ (the multi-indices), define $p_{\alpha}:=\prod_{i=1}^{N} p_{\alpha_{i}}\left(x_{i} ; x\right)$. The set $\left\{p_{\alpha}: \alpha \in \mathbb{N}_{0}^{N}\right\}$ is a basis for the polynomials for generic $\kappa$. The key property is that $T_{j} p_{n}\left(x_{i} ; x\right)=0$ for $j \neq i$. From [1] (Section 10.3), we find

$$
\begin{aligned}
T_{i} p_{\alpha} & =\left(N \kappa+\alpha_{i}\right) p_{\alpha_{i}-1}\left(x_{i} ; x\right) \prod_{m \neq i} p_{\alpha_{m}}\left(x_{m} ; x\right) \\
& +\kappa \sum_{j \neq i} \sum_{m=0}^{\alpha_{j}-1}\left(p_{\alpha_{i}+\alpha_{j}-1-m}\left(x_{i} ; x\right) p_{m}\left(x_{j} ; x\right)\right. \\
& \left.-p_{m}\left(x_{i} ; x\right) p_{\alpha_{i}+\alpha_{j}-1-m}\left(x_{j} ; x\right)\right) \times \prod_{n \neq i, j} p_{\alpha_{n}}\left(x_{n} ; x\right),
\end{aligned}
$$

if $\alpha_{i}>0$, and $T_{i} p_{\alpha}=0$ if $\alpha_{i}=0$.

Set up a symbolic calculus by letting $p_{j}^{n}$ denote $p_{n}\left(x_{j} ; x\right)$; more formally, define a linear isomorphism from ordinary polynomials to polynomials in the variables $\left\{p_{1}, \ldots, p_{N}\right\}$ :

$$
\Psi p_{\alpha}=p_{1}^{\alpha_{1}} p_{2}^{\alpha_{2}} \cdots p_{N}^{\alpha_{N}}, \alpha \in \mathbb{N}_{0}^{N}
$$


extended by linearity. Thus, $\Psi \sum_{n=0}^{\infty} p_{n}\left(x_{i} ; x\right) r^{n}=\left(1-p_{i} r\right)^{-1}$. In this form, the action of $T_{i}$ (technically $\left.\Psi T_{i} \Psi^{-1}\right)$ on a function of $\left(p_{1}, \ldots, p_{N}\right)$ is given by

$$
\begin{aligned}
T_{i} f(p) & =\frac{\partial f}{\partial p_{i}}+N \kappa \frac{f-\left(p_{i} \rightarrow 0\right) f}{p_{i}} \\
& +\kappa \sum_{j=1, j \neq i}^{N} \frac{\left(p_{i} \rightarrow p_{j}\right) f+\left(p_{j} \rightarrow p_{i}\right) f-f-\left(p_{j} \longleftrightarrow p_{i}\right) f}{p_{i}-p_{j}} .
\end{aligned}
$$

The operators $\left(p_{i} \rightarrow 0\right)$ and $\left(p_{i} \rightarrow p_{j}\right)$ replace $p_{i}$ by 0 and $p_{j}$ respectively, while $\left(p_{j} \longleftrightarrow p_{i}\right)$ is the transposition. It suffices to examine the effect of the formula on monomials $p_{1}^{\alpha_{1}} p_{2}^{\alpha_{2}} \ldots p_{N}^{\alpha_{N}}$ and for $i=1$. The first two terms produce $\left(\alpha_{1}+N \kappa\right)$ if $\alpha_{1}>0$, else 0 . In the sum, the (typical) term for $j=2$ is $\left(p_{1}^{\alpha_{1}+\alpha_{2}}+p_{2}^{\alpha_{1}+\alpha_{2}}-p_{1}^{\alpha_{1}} p_{2}^{\alpha_{2}}-p_{1}^{\alpha_{2}} p_{2}^{\alpha_{1}}\right) \prod_{m=3}^{N} p_{m}^{\alpha_{m}} /\left(p_{1}-p_{2}\right)$. A simple calculation shows that this is the image under $\Psi$ of the corresponding term in Equation (1). This method was used in [3] to find planar harmonics of type $B$ (the group generated by sign-changes and permutation of coordinates).

From here on, we will be concerned with polynomials in $p_{1}, p_{2}$, that is, exactly the set of polynomials annihilated by $T_{j}$ for $2<j \leq N$. Set $p_{i, j}:=p_{i}\left(x_{1} ; x\right) p_{j}\left(x_{2} ; x\right)$ so that $\Psi p_{i, j}=p_{1}^{i} p_{2}^{j}$. For each degree $\geq 1$, there are two independent harmonic polynomials, that is, $\left(T_{1}^{2}+T_{2}^{2}\right) f=0$, and a convenient orthogonal decomposition is by the action of $(1,2)$; symmetric: $(1,2) f=f$, and antisymmetric: $(1,2) f=-f$, to be designated by + and - superscripts, respectively. We use the operators $T_{1}+T_{2}$ and $T_{1}-T_{2}$ (note $\left.\left(T_{1}^{2}+T_{2}^{2}\right)=\frac{1}{2}\left(T_{1}+T_{2}\right)^{2}+\frac{1}{2}\left(T_{1}-T_{2}\right)^{2}\right)$. The harmonic polynomials will be expressed in the basis functions (symmetric) $\phi_{n j}$ and (antisymmetric) $\psi_{n j}$ with generating functions $u_{1}, u_{2}$ (and $s:=\frac{1}{2}\left(z+z^{-1}\right)$ ) given by

$$
\begin{aligned}
w_{1}:=\left(1-z t p_{1}\right)^{-1}\left(1-z^{-1} t p_{2}\right)^{-1}, \\
w_{2}:=\left(1-z^{-1} t p_{1}\right)^{-1}\left(1-z t p_{2}\right)^{-1}, \\
u_{1}:=\frac{1}{2}\left(w_{1}+w_{2}\right)=\frac{1-s t\left(p_{1}+p_{2}\right)+t^{2} p_{1} p_{2}}{\left(1-2 s t p_{1}+t^{2} p_{1}^{2}\right)\left(1-2 s t p_{2}+t^{2} p_{2}^{2}\right)^{\prime}} \\
u_{2}:=\left(z-\frac{1}{z}\right)^{-1}\left(w_{1}-w_{2}\right)=\frac{t\left(p_{1}-p_{2}\right)}{\left(1-2 s t p_{1}+t^{2} p_{1}^{2}\right)\left(1-2 s t p_{2}+t^{2} p_{2}^{2}\right)}, \\
u_{1}=\sum_{n=0}^{\infty} t^{n} \sum_{j=0}^{n} s^{j} \phi_{n j}, \\
u_{2}=\sum_{n=1}^{\infty} t^{n} \sum_{j=0}^{n} s^{j} \psi_{n j} .
\end{aligned}
$$

There are parity conditions: $\phi_{n j} \neq 0$ implies $j \equiv n \bmod 2$ and $\psi_{n j} \neq 0$ implies $j \equiv(n-1) \bmod 2$. These are formal power series and convergence is not important (but is assured if $\left.\max (|z t|,|t / z|)<\left(\max _{i}\left|x_{i}\right|\right)^{-1}\right)$. The polynomials $\phi_{n j}$ and $\psi_{n j}$ are homogeneous of degree $n$. The ordinary harmonic polynomials arise for $\kappa=0$; however, the following formulae remain complicated for this specialization. The polynomial $p_{i, j}$ reduces to $x_{1}^{i} x_{2}^{j}$. By using complex variables, the derivation goes quickly: the real and imaginary parts of $\left\{\left(x_{1}+x_{2}\right)+\mathrm{i}\left(x_{1}-x_{2}\right)\right\}^{n}$ are the symmetric and antisymmetric harmonic homogenous polynomials of degree $n$, respectively. The expression expands to

$$
\sum_{j=0}^{n}\left(\begin{array}{c}
n \\
j
\end{array}\right) x_{1}^{n-j} x_{2}^{j}(1+\mathrm{i})^{n-j}(1-\mathrm{i})^{j}=\sum_{j=0}^{n}\left(\begin{array}{c}
n \\
j
\end{array}\right) x_{1}^{n-j} x_{2}^{j}(1+\mathrm{i})^{n-2 j} 2^{j}
$$


The formulae for the desired polynomials can be obtained from the equation $(1+\mathrm{i})^{4 m+k}=(-4)^{m}$, $(-4)^{m}(1+\mathrm{i}),(-1)^{m} 2^{2 m+1} \mathrm{i},(-1)^{m+1} 2^{2 m+1}(1-\mathrm{i})$ for $k=0,1,2,3$ respectively. The noticeable difference between even and odd degrees $n$ will appear again in the general $\kappa$ case.

The following expressions are derived in Section 5: (the Pochhammer symbol is given by $(v)_{0}:=1$ and $(v)_{m}:=\prod_{j=1}^{m}(v+j-1)$ for $\left.m \geq 1\right)$ for $0 \leq j \leq\left\lfloor\frac{n}{2}\right\rfloor$

$$
\begin{aligned}
\phi_{n, n-2 j} & =2^{n-1-2 j} \sum_{i=0}^{j} \frac{(n+1-2 j)_{2 i}}{i !(1-n+2 j-2 i)_{i}}\left(p_{n-j+i, j-i}+p_{j-i, n-j+i}\right) \\
& =2^{n-1-2 j}\left(p_{n-j, j}+p_{j, n-j}\right)+2^{n-1-2 j} \\
& \times \sum_{i=1}^{j}(n-2 j+2 i) \frac{(n+1-2 j)_{i-1}}{i !}(-1)^{i}\left(p_{n-j+i, j-i}+p_{j-i, n-j+i}\right) ;
\end{aligned}
$$

and, for $0 \leq j \leq\left\lfloor\frac{n-1}{2}\right\rfloor$,

$$
\psi_{n, n-1-2 j}=2^{n-1-2 j} \sum_{i=0}^{j} \frac{(n-2 j)_{i}}{i !}(-1)^{i}\left(p_{n-j+i, j-i}-p_{j-i, n-j+i}\right) .
$$

The reason for the use of this basis is that the actions of $T_{1}+T_{2}$ and $T_{1}-T_{2}$ have relatively simple expressions. It is easy to verify that ( $\operatorname{set} \partial_{v}:=\frac{\partial}{\partial v}$ for a variable $v$ ):

$$
\begin{aligned}
& \partial_{p_{1}} w_{1}=z t\left(w_{1}+\frac{t}{2} \partial_{t} w_{1}\right)+\frac{z^{2} t}{2} \partial_{z} w_{1}, \partial_{p_{2}} w_{1}=\frac{t}{z}\left(w_{1}+\frac{t}{2} \partial_{t} w_{1}\right)-\frac{t}{2} \partial_{z} w_{1} \\
& \partial_{p_{1}} w_{2}=\frac{t}{z}\left(w_{2}+\frac{t}{2} \partial_{t} w_{2}\right)-\frac{t}{2} \partial_{z} w_{2}, \partial_{p_{2}} w_{2}=z t\left(w_{2}+\frac{t}{2} \partial_{t} w_{2}\right)+\frac{z^{2} t}{2} \partial_{z} w_{2}
\end{aligned}
$$

After some calculations involving $\frac{\partial}{\partial z}=\frac{1}{2}\left(1-z^{-2}\right) \frac{\partial}{\partial s}$, we obtain

$$
\begin{aligned}
& \left(\partial_{p_{1}}+\partial_{p_{2}}\right) u_{1}=2 s t u_{1}+s t^{2} \partial_{t} u_{1}+\left(s^{2}-1\right) t \partial_{s} u_{1}, \\
& \left(\partial_{p_{1}}-\partial_{p_{2}}\right) u_{1}=\left(3 s^{2}-2\right) t u_{2}+\left(s^{2}-1\right) t^{2} \partial_{t} u_{2}+s\left(s^{2}-1\right) t \partial_{s} u_{2} \\
& \left(\partial_{p_{1}}+\partial_{p_{2}}\right) u_{2}=3 s t u_{2}+s t^{2} \partial_{t} u_{2}+\left(s^{2}-1\right) t \partial_{s} u_{2} \\
& \left(\partial_{p_{1}}-\partial_{p_{2}}\right) u_{2}=2 t u_{1}+t^{2} \partial_{t} u_{1}+s t \partial_{s} u_{1} .
\end{aligned}
$$

Applying

$$
\begin{aligned}
T_{1}+T_{2}-\partial_{p_{1}}-\partial_{p_{2}} & =N \kappa \frac{1-\left(p_{1} \rightarrow 0\right)}{p_{1}}+N \kappa \frac{1-\left(p_{2} \rightarrow 0\right)}{p_{2}} \\
T_{1}-T_{2}-\partial_{p_{1}}+\partial_{p_{2}} & =N \kappa \frac{1-\left(p_{1} \rightarrow 0\right)}{p_{1}}-N \kappa \frac{1-\left(p_{2} \rightarrow 0\right)}{p_{2}} \\
& +2 \kappa \frac{\left(p_{1} \rightarrow p_{2}\right)+\left(p_{2} \rightarrow p_{1}\right)-1-\left(p_{1} \longleftrightarrow p_{2}\right)}{p_{1}-p_{2}}
\end{aligned}
$$


to $u_{1}$ and $u_{2}$ yields

$$
\begin{aligned}
& \left(T_{1}+T_{2}-\partial_{p_{1}}-\partial_{p_{2}}\right) u_{1}=2 s t N \kappa u_{1} \\
& \left(T_{1}-T_{2}-\partial_{p_{1}}+\partial_{p_{2}}\right) u_{1}=2 t^{2}\left(N\left(s^{2}-1\right)+1\right) \kappa u_{2} \\
& \left(T_{1}+T_{2}-\partial_{p_{1}}-\partial_{p_{2}}\right) u_{2}=2 t s N \kappa u_{2} \\
& \left(T_{1}-T_{2}-\partial_{p_{1}}+\partial_{p_{2}}\right) u_{2}=2 t N \kappa u_{1} .
\end{aligned}
$$

Applying these to the generating functions results in

$$
\begin{aligned}
\left(T_{1}+T_{2}\right) \phi_{n j} & =-(j+1) \phi_{n-1, j+1}+(2 N \kappa+n+j) \phi_{n-1, j-1}, \\
\left(T_{1}-T_{2}\right) \phi_{n, j} & =-(2 N \kappa-2 \kappa+n+j+1) \psi_{n-1, j}+(2 N \kappa+n+j) \psi_{n-1, j-2}, \\
\left(T_{1}+T_{2}\right) \psi_{n j} & =-(j+1) \psi_{n-1 . j+1}+(2 N \kappa+n+j+1) \psi_{n-1, j-1}, \\
\left(T_{1}-T_{2}\right) \psi_{n j} & =(2 N \kappa+n+j+1) \phi_{n-1, j} .
\end{aligned}
$$

We will state the expressions for the harmonic polynomials before their derivations; however, it is necessary to define two families of polynomials via three-term relations. The motivation comes later.

Definition 2. For $n=0,1,2, \ldots$ define two families of polynomials by

$$
\begin{aligned}
& g_{0}^{o}(v)=1, g_{n+1}^{o}(v)=(v+3 n+1) g_{n}^{o}(v)-n(2 n-1) g_{n-1}^{o}(v), \\
& g_{0}^{e}(v)=1, g_{n+1}^{e}(v)=(v+3 n+2) g_{n}^{e}(v)-n(2 n+1) g_{n-1}^{e}(v) .
\end{aligned}
$$

The first few polynomials are

$$
\begin{aligned}
& g_{1}^{o}(v)=v+1 \\
& g_{2}^{o}(v)=v^{2}+5 v+3 \\
& g_{3}^{o}(v)=v^{3}+12 v^{2}+32 v+15
\end{aligned}
$$

and

$$
\begin{aligned}
& g_{1}^{e}(v)=v+2 \\
& g_{2}^{e}(v)=v^{2}+7 v+7 \\
& g_{3}^{e}(v)=v^{3}+15 v^{2}+53 v+36
\end{aligned}
$$

The three-term recurrences and Favard's theorem imply that both $\left\{g_{n}^{0}: n \geq 0\right\}$ and $\left\{g_{n}^{e}: n \geq 0\right\}$ are families of orthogonal polynomials for some (unknown) measures supported on $(-\infty, 0)$, but they do not appear to be of Askey tableau type. There are relations between the two families, stated without proof: $g_{n}^{e}(v)=g_{n}^{o}(v)-g_{n-1}^{o}(v)$ and $v g_{n-1}^{o}(v)=g_{n}^{e}(v)-(2 n-1) g_{n-1}^{e}(v)$. 
Definition 3. For $m=0,1,2, \ldots$ let

$$
\begin{aligned}
h_{2 m+1}^{-} & :=\sum_{j=0}^{m} 2^{-j} \frac{g_{j}^{o}(N \kappa-\kappa+m)}{(N \kappa+m+2)_{j}} \psi_{2 m+1,2 j,} \\
h_{2 m}^{-} & :=\sum_{j=0}^{m-1} 2^{-j} \frac{g_{j}^{e}(N \kappa-\kappa+m)}{(N \kappa+m+2)_{j}} \psi_{2 m, 2 j+1,} \\
h_{2 m+1}^{+} & :=\sum_{j=0}^{m} 2^{-j} \frac{g_{j}^{e}(N \kappa-\kappa+m+1)}{(N \kappa+m+2)_{j}} \phi_{2 m+1,2 j+1,} \\
h_{2 m}^{+} & :=\sum_{j=0}^{m} 2^{-j} \frac{g_{j}^{o}(N \kappa-\kappa+m)}{(N \kappa+m+1)_{j}} \phi_{2 m, 2 j ;}
\end{aligned}
$$

each $h_{n}^{ \pm}$is homogeneous of degree $n$.

First, we show that the antisymmetric polynomials $h_{n}^{-}$are harmonic. We will use the relations (3) and (5) to produce symmetric harmonic polynomials from the antisymmetric ones. Combining Equations (3)-(5) obtains

$$
\begin{aligned}
& \left(\left(T_{1}+T_{2}\right)^{2}+\left(T_{1}-T_{2}\right)^{2}\right) \psi_{n j} \\
& =(j+1)(j+2) \psi_{n-2, j+2}-(2 N \kappa+n+j+1)(2 N \kappa-2 \kappa+n+3 j+1) \psi_{n-2, j} \\
& +2(2 N \kappa+n+j+1)(2 N \kappa+n-j+1) \psi_{n-2, j-2} .
\end{aligned}
$$

Suppose $h_{n}^{-}=\sum_{j=0}^{\lfloor(n-1) / 2\rfloor} c_{n-1-2 j} \psi_{n, n-1-2 j}$ is harmonic; then, the coefficient of $\psi_{n-2, n-2 j-1}$ in $2 \Delta_{\kappa} h_{n}^{-}$is

$$
\begin{aligned}
0 & =8(N \kappa+n-j+1)(N \kappa+n-j) c_{n+1-2 j} \\
& -4(N \kappa-\kappa+2 n-1-3 j)(N \kappa+n-j) c_{n-1-2 j} \\
& +(n-2 j-2)(n-2 j-1) c_{n-3-2 j} .
\end{aligned}
$$

The range of $j$ is derived from the inequality $0 \leq n-2 j-1 \leq n-3$, which is $1 \leq j \leq \frac{n-1}{2}$. Two sets of formulae arise depending on the parity of $n$. The equations are considered as recurrences.

Suppose $n=2 m+1$ then the starting point is for $j=m$

$$
8(N \kappa+m+2)(N \kappa+m+1) c_{2}-4(N \kappa-\kappa+m+1)(N \kappa+m+1) c_{0}=0
$$

thus

$$
c_{2}=\frac{1}{2} \frac{N \kappa-\kappa+m+1}{N \kappa+m+2} c_{0} .
$$

Set $j=m-i$ to obtain

$$
\begin{aligned}
& c_{2 i+2} \\
& =\frac{1}{2} \frac{(N \kappa-\kappa+m+1+3 i)}{N \kappa+m+2+i} c_{2 i}-\frac{1}{4} \frac{(2 i-1) i}{(N \kappa+m+2+i)(N \kappa+m+1+i)} c_{2 i-2} .
\end{aligned}
$$

To simplify the recurrences, let $\gamma_{i}^{o}=2^{i} c_{2 i}(N \kappa+m+2)_{i} / c_{0}$; then, $\gamma_{0}^{o}=1$ and

$$
\gamma_{i+1}^{o}=(N \kappa-\kappa+m+1+3 i) \gamma_{i}^{o}-i(2 i-1) \gamma_{i-1}^{o},
$$

which agrees with the recurrence for $g_{i}^{o}$ with $v=N \kappa-\kappa+m$. 
Thus, the antisymmetric harmonic polynomial of degree $2 m+1$ (normalized by $c_{0}=1$ ) is

$$
h_{2 m+1}^{-}=\sum_{j=0}^{m} 2^{-j} \frac{g_{j}^{o}(N \kappa-\kappa+m)}{(N \kappa+m+2)_{j}} \psi_{2 m+1,2 j} .
$$

Suppose $n=2 m$; then, the starting point is for $j=m-1$

$$
8(N \kappa+m+2)(N \kappa+m+1) c_{3}-4(N \kappa-\kappa+m+2)(N \kappa+m+1) c_{1}=0,
$$

so that

$$
c_{3}=\frac{1}{2} \frac{N \kappa-\kappa+m+2}{N \kappa+m+2} c_{1}
$$

Set $j=m-1-i$ to obtain

$$
\begin{aligned}
& c_{2 i+3} \\
& =\frac{1}{2} \frac{(N \kappa-\kappa+m+2+3 i)}{N \kappa+m+2+i} c_{2 i+1}-\frac{1}{4} \frac{(2 i+1) i}{(N \kappa+m+2+i)(N \kappa+m+1+i)} c_{2 i-1} .
\end{aligned}
$$

Similarly to the previous calculation, let $\gamma_{i}^{e}:=2^{i} c_{2 i+1}(N \kappa+m+2)_{i} / c_{1}$; then, $\gamma_{o}^{e}=1$ and

$$
\gamma_{i+1}^{e}=(N \kappa-\kappa+m+2+3 i) \gamma_{i}^{e}-i(2 i+1) \gamma_{i-1}^{e} .
$$

This agrees with the recurrence for $g_{i}^{e}$ with $v=N \kappa-\kappa+m$. Thus, the antisymmetric harmonic polynomial of degree $2 m$ (normalized by $c_{1}=1$ ) is

$$
h_{2 m}^{-}=\sum_{j=0}^{m-1} 2^{-j} \frac{g_{j}^{e}(N \kappa-\kappa+m)}{(N \kappa+m+2)_{j}} \psi_{2 m, 2 j+1} .
$$

Applying $\left(T_{1}-T_{2}\right)$ to a harmonic polynomial clearly produces another harmonic polynomial; thus, by Equation (5):

$$
\begin{aligned}
& \frac{1}{2(N \kappa+m+1)}\left(T_{1}-T_{2}\right) h_{2 m+1}^{-} \\
& =\sum_{j=0}^{m} 2^{-j} \frac{g_{j}^{o}(N \kappa-\kappa+m)}{(N \kappa+m+1)_{j+1}}(N \kappa+m+j+1) \phi_{2 m, 2 j} \\
& =\sum_{j=0}^{m} 2^{-j} \frac{g_{j}^{o}(N \kappa-\kappa+m)}{(N \kappa+m+1)_{j}} \phi_{2 m, 2 j}=h_{2 m}^{+}
\end{aligned}
$$

and

$$
\begin{aligned}
& \frac{1}{2(N \kappa+m+1)}\left(T_{1}-T_{2}\right) h_{2 m}^{-} \\
& =\sum_{j=0}^{m-1} 2^{-j} \frac{g_{j}^{e}(N \kappa-\kappa+m)}{(N \kappa+m+1)_{j+1}}(N \kappa+m+j+1) \phi_{2 m-1,2 j+1} \\
& =\sum_{j=0}^{m-1} 2^{-j} \frac{g_{j}^{e}(N \kappa-\kappa+m)}{(N \kappa+m+1)_{j}} \phi_{2 m-1,2 j+1}=h_{2 m-1}^{+} .
\end{aligned}
$$

We have proven: 
Proposition 1. The polynomials $h_{n}^{+}$and $h_{n}^{-}$are harmonic.

For use in the sequel, we find expressions for $T_{1} \pm T_{2}$ applied to $h_{n}^{+}$and $h_{n}^{-}$.

Proposition 2. The actions of $T_{1} \pm T_{2}$ on the antisymmetric polynomials $h_{n}^{-}$are

$$
\begin{aligned}
\left(T_{1}-T_{2}\right) h_{2 m+1}^{-} & =2(N \kappa+m+1) h_{2 m^{\prime}}^{+} \\
\left(T_{1}-T_{2}\right) h_{2 m}^{-} & =2(N \kappa+m+1) h_{2 m-1^{\prime}}^{+} \\
\left(T_{1}+T_{2}\right) h_{2 m+1}^{-} & =(N \kappa-\kappa+m) h_{2 m^{\prime}}^{-} \\
\left(T_{1}+T_{2}\right) h_{2 m}^{-} & =2(N \kappa+m+1) h_{2 m-1^{\prime}}^{-}
\end{aligned}
$$

and the actions on the symmetric polynomials $h_{n}^{+}$are

$$
\begin{aligned}
\left(T_{1}-T_{2}\right) h_{2 m+1}^{+} & =-(N \kappa-\kappa+m) h_{2 m^{\prime}}^{-} \\
\left(T_{1}-T_{2}\right) h_{2 m}^{+} & =-(N \kappa-\kappa+m) h_{2 m-1^{\prime}}^{-} \\
\left(T_{1}+T_{2}\right) h_{2 m+1}^{+} & =2(N \kappa+m+1) h_{2 m^{\prime}}^{+} \\
\left(T_{1}+T_{2}\right) h_{2 m}^{+} & =(N \kappa-\kappa+m) h_{2 m-1}^{+} .
\end{aligned}
$$

Since the resulting polynomials are harmonic, it suffices to consider just one term in their expansions. The coefficients of the lowest index term $\left(\phi_{2 m-1,1}, \phi_{2 m, 0}, \psi_{2 m-1,0}, \psi_{2 m, 1}\right.$ for $h_{2 m-1}^{+}, h_{2 m}^{+}, h_{2 m-1}^{-}, h_{2 m}^{-}$, respectively) on the right sides arise from at most two terms on the left. The details are in Section 5.

\section{Inner Products and Structure Constants}

Let $\mu$ denote the Gaussian measure $(2 \pi)^{-N / 2} e^{-|x|^{2} / 2} \mathrm{~d} x$ on $\mathbb{R}^{N}$, (where $d x$ is the Lebesgue measure), and let $m$ denote the normalized surface measure on $S_{N-1}:=\left\{x \in \mathbb{R}^{N}:|x|=1\right\}$. The weight function is $w_{\kappa}(x):=\prod_{1 \leq i<j \leq N}\left|x_{i}-x_{j}\right|^{2 \kappa}$. The constants $c_{\kappa}$ and $c_{k}^{\prime}$ are defined by $c_{\mathcal{K}} \int_{\mathbb{R}^{N}} w_{\kappa} \mathrm{d} \mu=1$ and $c_{\kappa}^{\prime} \int_{S_{N-1}} w_{\kappa} \mathrm{d} m=1$. It is known (the Macdonald-Mehta-Selberg integral) that $c_{\kappa}=\prod_{j=2}^{N}\left(\frac{\Gamma(\kappa+1)}{\Gamma(j \kappa+1)}\right)$; this integral appeared in the probability distribution of eigenvalues of random Hermitian matrices in Mehta's investigations, and conjectures by Macdonald for integrals related to root systems-a proof using an integral of Selberg's was eventually found (see Askey [4]). There is an elegant proof applying to all finite reflection groups due to Etingof [5]. There are three inner products for polynomials associated with $\Delta_{\kappa}$. For polynomials $f, g$ define

1. $\langle f, g\rangle_{\mathcal{K}}:=\left.f\left(T_{1}, \ldots, T_{N}\right) g(x)\right|_{x=0}$ (evaluated at $\left.x=0\right)$;

2. $\langle f, g\rangle_{G}:=c_{\mathcal{K}} \int_{\mathbb{R}^{N}} f g w_{\mathcal{K}} \mathrm{d} \mu$, the Gaussian inner product;

3. $\langle f, g\rangle_{S}:=c_{\kappa}^{\prime} \int_{S_{N-1}} f g w_{\kappa} \mathrm{d} m$.

The details can be found in [1] (Chapter 7.2). There are important relations among them: $\langle f, g\rangle_{\kappa}=\left\langle e^{-\Delta_{\kappa} / 2} f, e^{-\Delta_{\kappa} / 2} g\right\rangle_{G}$ (note that the series $\sum_{j \geq 0} \frac{1}{j !}\left(-\frac{\Delta_{\kappa}}{2}\right)^{j} f$ terminates for any polynomial $f$ ) and if $f$ is homogeneous of degree $2 n$, then

$$
\int_{\mathbb{R}^{N}} f w_{\mathcal{K}} \mathrm{d} \mu=2^{n+N(N-1) \kappa / 2} \frac{\Gamma\left(\frac{N}{2}((N-1) \kappa+1)+n\right)}{\Gamma\left(\frac{N}{2}\right)} \int_{S_{N-1}} f w_{\mathcal{K}} \mathrm{d} m .
$$


Specialized to $f=1$, this shows that

$$
c_{\mathcal{K}}^{\prime}=2^{N(N-1) \kappa / 2} \frac{\Gamma\left(\frac{N}{2}((N-1) \kappa+1)\right)}{\Gamma\left(\frac{N}{2}\right)} c_{\kappa},
$$

and thus

$$
c_{\mathcal{K}} \int_{\mathbb{R}^{N}} f w_{\kappa} \mathrm{d} \mu=2^{N(N-1) \kappa / 2}\left(\frac{N}{2}((N-1) \kappa+1)\right)_{n} c_{\mathcal{K}}^{\prime} \int_{S_{N-1}} f w_{\kappa} \mathrm{d} m .
$$

As a consequence, if $f$ and $g$ are harmonic and homogeneous of degrees $m, n$, respectively, then

$$
\langle f, g\rangle_{\kappa}=\langle f, g\rangle_{G}=2^{n}\left(\frac{N}{2}((N-1) \kappa+1)\right)_{n} \delta_{m n}\langle f, g\rangle_{S} .
$$

It is a fundamental result that $\operatorname{deg} f \neq \operatorname{deg} g$ implies $\langle f, g\rangle_{S}=0$.

To find $\langle f, f\rangle_{\kappa}$ for the harmonic polynomials $h_{n}^{+}, h_{n}^{-}$, we will need the values of $\phi_{n j}$ and $\psi_{n j}$ at $x=\left(x_{1}, x_{2}, 0, \ldots, 0\right)$. In terms of the generating functions,

$$
\Psi^{-1}\left(1-r p_{1}\right)^{-1}=\Psi^{-1} \sum_{n=0}^{\infty} p_{1}^{n} r^{n}=\left(1-r x_{1}\right)^{-\kappa-1}\left(1-r x_{2}\right)^{-\kappa}
$$

thus

$$
\begin{aligned}
& w_{1}(x):=\left(1-z t x_{1}\right)^{-1-\kappa}\left(1-z t x_{2}\right)^{-\kappa}\left(1-z^{-1} t x_{2}\right)^{-1-\kappa}\left(1-z^{-1} t x_{1}\right)^{-\kappa} \\
& w_{2}(x):=\left(1-z t x_{2}\right)^{-1-\kappa}\left(1-z t x_{1}\right)^{-\kappa}\left(1-z^{-1} t x_{1}\right)^{-1-\kappa}\left(1-z^{-1} t x_{2}\right)^{-\kappa}
\end{aligned}
$$

and

$$
\begin{aligned}
u_{1}(x) & =\frac{1}{2}\left(w_{1}(x)+w_{2}(x)\right) \\
& =\frac{\left(1-z t x_{2}\right)\left(1-z^{-1} t x_{1}\right)+\left(1-z t x_{1}\right)\left(1-z^{-1} t x_{2}\right)}{2\left\{\left(1-2 s t x_{1}+x_{1}^{2} t^{2}\right)\left(1-2 s t x_{2}+x_{2}^{2} t^{2}\right)\right\}^{\kappa+1}} \\
& =\frac{1-\left(x_{1}+x_{2}\right) s t+x_{1} x_{2} t^{2}}{\left\{\left(1-2 s t x_{1}+x_{1}^{2} t^{2}\right)\left(1-2 s t x_{2}+x_{2}^{2} t^{2}\right)\right\}^{\kappa+1}}, \\
u_{2}(x) & =\left(z-\frac{1}{z}\right)^{-1}\left(w_{1}(x)-w_{2}(x)\right) \\
& =\frac{\left(x_{1}-x_{2}\right) t}{\left\{\left(1-2 s t x_{1}+x_{1}^{2} t^{2}\right)\left(1-2 s t x_{2}+x_{2}^{2} t^{2}\right)\right\}^{\kappa+1}}
\end{aligned}
$$

because $\left(z-z^{-1}\right)^{-1}\left\{\left(1-z t x_{2}\right)\left(1-z^{-1} t x_{1}\right)-\left(1-z t x_{1}\right)\left(1-z^{-1} t x_{2}\right)\right\}=\left(x_{1}-x_{2}\right) t$. Thus, $\phi_{n j}(x)=$ coef $\left(u_{1}(x), t^{n} s^{j}\right)$ and $\psi_{n j}(x)=\operatorname{coef}\left(u_{2}(x), t^{n} s^{j}\right)$.

By the $(1,2)$ symmetry (both $w_{\kappa}$ and $h_{n}^{+}$are invariant and $h_{n}^{-}$changes sign), the inner products $\left\langle h_{n}^{+}, h_{n}^{-}\right\rangle=0$. Next, we compute the pairing $\langle f, f\rangle_{\mathcal{K}}$ for the harmonic polynomials. Since they are annihilated by $T_{j}$ for $j>2$, these values are given by $f\left(T_{1}, T_{2}, 0, \ldots\right) f$. We use the harmonicity of $f$, that is, $\left(T_{1}-T_{2}\right)^{2} f=-\left(T_{1}+T_{2}\right)^{2} f$. The same relation holds when $f$ is replaced by $q\left(T_{1}, T_{2}\right) f$ for any polynomial $q$. Suppose $\operatorname{deg} f=n$ and express

$$
f\left(x_{1}, x_{2}, 0 \ldots\right)=\sum_{j=0}^{n} c_{j}\left(x_{1}+x_{2}\right)^{n-j}\left(x_{1}-x_{2}\right)^{j} .
$$


If $(1,2) f=f$, then $c_{j}=0$ for odd $j$ and

$$
\begin{aligned}
f\left(T_{1}, T_{2}, 0 \ldots\right) f & =\sum_{j=0}^{\lfloor n / 2\rfloor} c_{2 j}\left(T_{1}+T_{2}\right)^{n-2 j}\left(T_{1}-T_{2}\right)^{2 j} f \\
& =\sum_{j=0}^{\lfloor n / 2\rfloor} c_{2 j}(-1)^{j}\left(T_{1}+T_{2}\right)^{n} f .
\end{aligned}
$$

Set $x_{1}=1+\mathrm{i}, x_{2}=1-\mathrm{i}$, and then

$$
f(x)=\sum_{j=0}^{\lfloor n / 2\rfloor} c_{2 j} 2^{n-2 j}(2 \mathrm{i})^{2 j}=2^{n} \sum_{j=0}^{\lfloor n / 2\rfloor} c_{2 j}(-1)^{j} ;
$$

thus,

$$
f\left(T_{1}, T_{2}, 0 \ldots\right) f=2^{-n} f(1+\mathrm{i}, 1-\mathrm{i}, 0 \ldots)\left(T_{1}+T_{2}\right)^{n} f .
$$

Proceeding similarly for $(1,2) f=-f$, where $c_{j}=0$ for even $j$, we obtain

$$
\begin{aligned}
f\left(T_{1}, T_{2}, 0 \ldots\right) f & =\sum_{j=0}^{\lfloor(n-1) / 2\rfloor} c_{2 j+1}\left(T_{1}+T_{2}\right)^{n-1-2 j}\left(T_{1}-T_{2}\right)^{2 j+1} f \\
& =\sum_{j=0}^{\lfloor(n-1) / 2\rfloor} c_{2 j+1}(-1)^{j}\left(T_{1}-T_{2}\right)\left(T_{1}+T_{2}\right)^{n-1} f,
\end{aligned}
$$

and

$$
f(1+\mathrm{i}, 1-\mathrm{i}, 0 \ldots)=\sum_{j=0}^{\lfloor(n-1) / 2\rfloor} c_{2 j+1} 2^{n-1-2 j}(2 \mathrm{i})^{2 j+1}=\mathrm{i} 2^{n} \sum_{j=0}^{\lfloor(n-1) / 2\rfloor} c_{2 j+1}(-1)^{j} .
$$

Thus,

$$
f\left(T_{1}, T_{2}, 0 \ldots\right) f=-\mathrm{i} 2^{-n} f(1+\mathrm{i}, 1-\mathrm{i}, 0 \ldots)\left(T_{1}+T_{2}\right)^{n-1}\left(T_{1}-T_{2}\right) f .
$$

First, the symmetric case (by Proposition 2):

$$
\left(T_{1}+T_{2}\right)^{2} h_{2 m}^{+}=\left(T_{1}+T_{2}\right)(N \kappa-\kappa+m) h_{2 m-1}^{+}=2(N \kappa-\kappa+m)(N \kappa+m) h_{2 m-2}^{+}
$$

and it follows by induction that

$$
\begin{aligned}
\left(T_{1}+T_{2}\right)^{2 m} h_{2 m}^{+} & =2^{m}(N \kappa-\kappa+1)_{m}(N \kappa+1)_{m}, \\
\left(T_{1}+T_{2}\right)^{2 m+1} h_{2 m+1}^{+} & =2(N \kappa+m+1)\left(T_{1}+T_{2}\right)^{2 m} h_{2 m^{\prime}}^{+} \\
& =2^{m+1}(N \kappa-\kappa+1)_{m}(N \kappa+1)_{m+1} .
\end{aligned}
$$

For the antisymmetric case:

$$
\begin{aligned}
& \left(T_{1}+T_{2}\right)^{2 m-1}\left(T_{1}-T_{2}\right) h_{2 m}^{-} \\
& =2(N \kappa+m+1)\left(T_{1}+T_{2}\right)^{2 m-1} h_{2 m-1}^{+} \\
& =2^{m+1}(N \kappa+m+1)(N \kappa-\kappa+1)_{m-1}(N \kappa+1)_{m} \\
& =2^{m+1}(N \kappa-\kappa+1)_{m-1}(N \kappa+1)_{m+1}
\end{aligned}
$$


and

$$
\begin{aligned}
& \left(T_{1}+T_{2}\right)^{2 m}\left(T_{1}-T_{2}\right) h_{2 m+1}^{-} \\
& =2(N \kappa+m+1)\left(T_{1}+T_{2}\right)^{2 m} h_{2 m}^{+} \\
& =2^{m+1}(N \kappa+m+1)(N \kappa-\kappa+1)_{m}(N \kappa+1)_{m} \\
& =2^{m+1}(N \kappa-\kappa+1)_{m}(N \kappa+1)_{m+1} .
\end{aligned}
$$

The values $\phi_{n j}(1+\mathrm{i}, 1-\mathrm{i}, 0 \ldots)$ and $\psi_{n j}(1+\mathrm{i}, 1-\mathrm{i}, 0 \ldots)$ are found by computing the generating functions:

$$
u_{1}(1+\mathrm{i}, 1-\mathrm{i}, 0 \ldots)=\frac{1-2 s t+2 t^{2}}{\left(1-4 s t+8 s^{2} t^{2}-8 s t^{3}+4 t^{4}\right)^{\kappa+1}}
$$

(the term in the denominator is $\left.\left(1-2 s t+2 t^{2}\right)^{2}-4\left(1-s^{2}\right) t^{2}\right)$ and

$$
u_{2}(1+\mathrm{i}, 1-\mathrm{i}, 0 \ldots)=\frac{2 \mathrm{i} t}{\left(1-4 s t+8 s^{2} t^{2}-8 s t^{3}+4 t^{4}\right)^{\kappa+1}} .
$$

Definition 4. For $n=0,1,2, \ldots, 0 \leq j \leq\left\lfloor\frac{n}{2}\right\rfloor$ and parameters $\alpha, \beta$, let

$$
S(n, j ; \alpha, \beta)
$$

$$
:=\sum_{\ell=0}^{\lfloor n / 2\rfloor} \sum_{i=\max (0, \ell+j-\lfloor n / 2\rfloor)}^{\min (\ell, j)} \frac{(\alpha+1)_{\ell}(2 \alpha+\beta+2 \ell)_{n-2 \ell-j+i}}{i !(\ell-i) !(j-i) !(n-2 \ell-2 j+2 i) !}(-1)^{\ell+j} 2^{n-j+i} .
$$

Proposition 3. For $0 \leq j \leq\left\lfloor\frac{n}{2}\right\rfloor$,

$$
\begin{aligned}
\phi_{n, n-2 j}(1+\mathrm{i}, 1-\mathrm{i}, 0, \ldots) & =S(n, j ; \kappa, 1), \\
\psi_{n+1, n-2 j}(1+\mathrm{i}, 1-\mathrm{i}, 0, \ldots) & =2 \mathrm{i} S(n, j ; \kappa, 2) .
\end{aligned}
$$

The proof is in Proposition 4.

Thus,

$$
\begin{aligned}
\left\langle h_{2 m}^{+}, h_{2 m}^{+}\right\rangle_{\kappa} & =2^{-2 m} h_{2 m}^{+}(1+\mathrm{i}, 1-\mathrm{i}, 0 \ldots)\left(T_{1}+T_{2}\right)^{2 m} h_{2 m}^{+} \\
& =2^{-m} \sum_{j=0}^{m} 2^{-j} \frac{g_{j}^{o}(N \kappa-\kappa+m)}{(N \kappa+m+1)_{j}} S(2 m, m-j ; \kappa, 1) \\
& \times(N \kappa-\kappa+1)_{m}(N \kappa+1)_{m}, \\
\left\langle h_{2 m+1}^{+}, h_{2 m+1}^{+}\right\rangle_{\kappa} & =2^{-2 m-1} h_{2 m+1}^{+}(1+\mathrm{i}, 1-\mathrm{i}, 0 \ldots)\left(T_{1}+T_{2}\right)^{2 m+1} h_{2 m+1}^{+} \\
& =2^{-m} \sum_{j=0}^{m} 2^{-j} \frac{g_{j}^{e}(N \kappa-\kappa+m+1)}{(N \kappa+m+2)_{j}} S(2 m+1, m-j ; \kappa, 1) \\
& \times(N \kappa-\kappa+1)_{m}(N \kappa+1)_{m+1},
\end{aligned}
$$


and

$$
\begin{aligned}
\left\langle h_{2 m}^{-}, h_{2 m}^{-}\right\rangle_{\kappa} & =-\mathrm{i} 2^{-2 m} h_{2 m}^{-}(1+\mathrm{i}, 1-\mathrm{i}, 0 \ldots)\left(T_{1}+T_{2}\right)^{2 m-1}\left(T_{1}-T_{2}\right) h_{2 m}^{-} \\
& =2^{-m+2} \sum_{j=0}^{m-1} 2^{-j} \frac{g_{j}^{e}(N \kappa-\kappa+m)}{(N \kappa+m+2)_{j}} S(2 m, m-j-1 ; \kappa, 2) \\
& \times(N \kappa-\kappa+1)_{m-1}(N \kappa+1)_{m+1}, \\
\left\langle h_{2 m+1}^{-}, h_{2 m+1}^{-}\right\rangle_{\kappa} & =-\mathrm{i} 2^{-2 m} h_{2 m+1}^{-}(1+\mathrm{i}, 1-\mathrm{i}, 0 \ldots)\left(T_{1}+T_{2}\right)^{2 m}\left(T_{1}-T_{2}\right) h_{2 m+1}^{-} \\
& =2^{2-m} \sum_{j=0}^{m} 2^{-j} \frac{g_{j}^{o}(N \kappa-\kappa+m)}{(N \kappa+m+2)_{j}} S(2 m+1, m-j ; \kappa, 2) \\
& \times(N \kappa-\kappa+1)_{m}(N \kappa+1)_{m+1} .
\end{aligned}
$$

The values of $\left\langle h_{n}^{+}, h_{n}^{+}\right\rangle_{S}$ and $\left\langle h_{n}^{-}, h_{n}^{-}\right\rangle_{S}$ can now be found by Equation (6). The expressions are complicated due to the fact that sign-changes are not in the symmetry group.

\section{The Dirac Operator and Monogenic Polynomials}

We use the Clifford algebra $C \ell_{N}$ over $\mathbb{R}$ generated by $\left\{\mathbf{e}_{1}, \mathbf{e}_{2}, \mathbf{e}_{3}, \ldots, \mathbf{e}_{N}\right\}$ with relations $\mathbf{e}_{i}^{2}=-1$ (that is, negative signature) and $\mathbf{e}_{i} \mathbf{e}_{j}=-\mathbf{e}_{j} \mathbf{e}_{i}$ for $i \neq j$. The type- $A$ Dirac operator acting on polynomials in $x \in \mathbb{R}^{N}$ with coefficients in $C \ell_{N}$ is defined by

$$
\boldsymbol{D} f:=\sum_{i=1}^{N} \mathbf{e}_{i} T_{i} f
$$

this implies $\boldsymbol{D}^{2}=-\sum_{i=1}^{N} T_{i}^{2}=-\Delta_{\kappa}$. A polynomial $f$ is said to be monogenic if $\boldsymbol{D} f=0$. The situation where the underlying symmetry group is $\mathbb{Z}_{2}^{N}$ has been investigated by De Bie, Genest and Vinet $[6,7]$. The planar harmonic polynomials found in the previous sections can be used to construct monogenic polynomials. They are of the form $f_{n}=h_{n}^{+}+\varepsilon h_{n}^{-}$with $\varepsilon \in C \ell_{N}$. By construction $T_{i} f=0$ for all $i>2$. To fit with the formulae in Proposition 2, write $\mathbf{e}_{1} T_{1}+\mathbf{e}_{2} T_{2}=\frac{1}{2}\left(\mathbf{e}_{1}+\mathbf{e}_{2}\right)\left(T_{1}+T_{2}\right)+$ $\frac{1}{2}\left(\mathbf{e}_{1}-\mathbf{e}_{2}\right)\left(T_{1}-T_{2}\right)$. Even and odd $n$ are handled separately:

$$
\begin{aligned}
& \left(\mathbf{e}_{1} T_{1}+\mathbf{e}_{2} T_{2}\right)\left(h_{2 m+1}^{+}+\varepsilon h_{2 m+1}^{-}\right) \\
& =\left(\mathbf{e}_{1}+\mathbf{e}_{2}\right)(N \kappa+m+1) h_{2 m}^{+}-\frac{1}{2}\left(\mathbf{e}_{1}-\mathbf{e}_{2}\right)(N \kappa-\kappa+m) h_{2 m}^{-} \\
& +\left(\mathbf{e}_{1}-\mathbf{e}_{2}\right) \varepsilon(N \kappa+m+1) h_{2 m}^{+}+\frac{1}{2}\left(\mathbf{e}_{1}+\mathbf{e}_{2}\right) \varepsilon(N \kappa-\kappa+m) h_{2 m}^{-} ;
\end{aligned}
$$

the coefficients of $(N \kappa+m+1) h_{2 m}^{+}$and $\frac{1}{2}(N \kappa-\kappa+m) h_{2 m}^{-}$are $\left(\mathbf{e}_{1}+\mathbf{e}_{2}\right)+\left(\mathbf{e}_{1}-\mathbf{e}_{2}\right) \varepsilon$ and $-\left(\mathbf{e}_{1}-\mathbf{e}_{2}\right)+\left(\mathbf{e}_{1}+\mathbf{e}_{2}\right) \varepsilon$, respectively. Both of these vanish for $\varepsilon=\mathbf{e}_{1} \mathbf{e}_{2}$. Thus, $\boldsymbol{D}\left(h_{2 m+1}^{+}+\mathbf{e}_{1} \mathbf{e}_{2} h_{2 m+1}^{-}\right)=0$. Since $\boldsymbol{D}$ commutes with $T_{1}+T_{2}$, the polynomial $\left(T_{1}+T_{2}\right)\left(h_{2 m+1}^{+}+\mathbf{e}_{1} \mathbf{e}_{2} h_{2 m+1}^{-}\right)$is also monogenic, and $\left(T_{1}+T_{2}\right)\left(h_{2 m+1}^{+}+\mathbf{e}_{1} \mathbf{e}_{2} h_{2 m+1}^{-}\right)=2(N \kappa+m+1) h_{2 m}^{+}+\mathbf{e}_{1} \mathbf{e}_{2}(N \kappa-\kappa+m) h_{2 m}^{-}$. This proves

$$
\begin{aligned}
\boldsymbol{D}\left(h_{2 m+1}^{+}+\mathbf{e}_{1} \mathbf{e}_{2} h_{2 m+1}^{-}\right) & =0, \\
\boldsymbol{D}\left(h_{2 m}^{+}+\mathbf{e}_{1} \mathbf{e}_{2} \frac{N \kappa-\kappa+m}{2(N \kappa+m+1)} h_{2 m}^{-}\right) & =0 .
\end{aligned}
$$




\section{Derivations of Various Formulae}

This section contains the derivations of some of the formulae appearing in the paper. The formulae for $\phi_{n, j}$ and $\psi_{n, j}$ are found by means of the Chebyshev polynomials $T_{k}$ and $U_{k}$ (see [2] (Section 4.1):

$$
\begin{aligned}
u_{1} & =\frac{1}{2} \sum_{k=0}^{\infty} \sum_{m=0}^{\infty} t^{k+m} p_{1}^{k} p_{2}^{m}\left(z^{k-m}+z^{m-k}\right) \\
& =\frac{1}{2} \sum_{n=0}^{\infty} t^{n} \sum_{m=0}^{n} p_{1}^{n-m} p_{2}^{m}\left(z^{n-2 m}+z^{2 m-n}\right) \\
& =\sum_{n=0}^{\infty} t^{n} \sum_{m=0}^{n} p_{1}^{n-m} p_{2}^{m} \cos ((n-2 m) \theta) \\
& =\sum_{n=0}^{\infty} t^{n} \sum_{m=0}^{n} p_{1}^{n-m} p_{2}^{m} T_{|n-2 m|}(s)
\end{aligned}
$$

where $z$ is replaced by $e^{\mathrm{i} \theta}$ and thus $s=\cos \theta$. The last inner sum can be written as $\sum_{m=0}^{\lfloor n / 2\rfloor} \varepsilon_{n, m}\left(p_{1}^{n-m} p_{2}^{m}+p_{1}^{m} p_{2}^{n-m}\right) T_{n-2 m}(s)$, where $\varepsilon_{n, m}=1$ except $\varepsilon_{2 m, m}=\frac{1}{2}$. Then, use the expansion $T_{k}(s)=\sum_{j=0}^{\lfloor k / 2\rfloor} \frac{(-k)_{2 j}}{j !(1-k)_{j}} 2^{k-1-2 j} s^{k-2 j}$ for $k \geq 1$ and extract the coefficient of $s^{n-2 j}$ to determine $\phi_{n, n-2 j}$. Applying the same technique to $u_{2}$, we obtain

$$
\begin{aligned}
u_{2} & =\frac{1}{z-z^{-1}} \sum_{n=0}^{\infty} t^{n} \sum_{m=0}^{n} p_{1}^{n-m} p_{2}^{m}\left(z^{n-2 m}-z^{2 m-n}\right) \\
& =\sum_{n=0}^{\infty} t^{n} \sum_{m=0}^{n} p_{1}^{n-m} p_{2}^{m} \frac{\sin ((n-2 m) \theta)}{\sin \theta} \\
& =\sum_{n=0}^{\infty} t^{n} \sum_{m=0}^{\lfloor n / 2\rfloor}\left(p_{1}^{n-m} p_{2}^{m}-p_{1}^{m} p_{2}^{n-m}\right) \frac{\sin ((n-2 m) \theta)}{\sin \theta} \\
& =\sum_{n=0}^{\infty} t^{n} \sum_{m=0}^{\lfloor n / 2\rfloor}\left(p_{1}^{n-m} p_{2}^{m}-p_{1}^{m} p_{2}^{n-m}\right) U_{n-1-2 m}(s) .
\end{aligned}
$$

Then, extract the coefficient of $s^{n-1-2 j}$ by means of the expansion $U_{k}(s)=\sum_{j=0}^{\lfloor k / 2\rfloor} \frac{(-k)_{2 j}}{j !(-k)_{j}} 2^{k-2 j} s^{k-2 j}$ for $k>0$ to find $\psi_{n, n-1-2 j}$.

Proof. (of Proposition 2). The formulae for $\left(T_{1}-T_{2}\right) h_{n}^{-}$have already been proven. For $\left(T_{1}+T_{2}\right) h_{n}^{-}$, substitute $n=2 m$ and $j=1$ in Formula (4) to obtain coef $\left(\left(T_{1}+T_{2}\right) h_{2 m}^{-}, \psi_{2 m-1,0}\right)=(2 N \kappa+2 m+2)$ and thus $\left(T_{1}+T_{2}\right) h_{2 m}^{-}=2(N \kappa+m+1) h_{2 m-1}^{-}$. Next, substitute $n=2 m+1$ and $j=0,2$ in Formula (4) to show that

$$
\begin{aligned}
& \operatorname{coef}\left(\left(T_{1}+T_{2}\right) h_{2 m+1}^{-}, \psi_{2 m, 1}\right) \\
& =-\operatorname{coef}\left(h_{2 m+1}^{-}, \psi_{2 m+1,0}\right)+(2 N \kappa+2 m+4) \operatorname{coef}\left(h_{2 m+1}^{-}, \psi_{2 m+1,2}\right) \\
& =-1+(N \kappa+m+2) \frac{g_{1}^{o}(N \kappa-\kappa+m)}{N \kappa+m+2}=N \kappa-\kappa+m .
\end{aligned}
$$

For $\left(T_{1}+T_{2}\right) h_{n}^{+}$, substitute $n=2 m+1, j=1$ in Formula (2) to show

$$
\begin{aligned}
\operatorname{coef}\left(\left(T_{1}+T_{2}\right) h_{2 m+1}^{+}, \phi_{2 m, 0}\right) & =(2 N \kappa+2 m+2) \operatorname{coef}\left(h_{2 m+1}^{+}, \phi_{2 m+1,1}\right) \\
& =2(N \kappa+m+1) .
\end{aligned}
$$


Next, substitute $n=2 m, j=0,2$ in Formula (2) to show that

$$
\begin{aligned}
& \operatorname{coef}\left(\left(T_{1}+T_{2}\right) h_{2 m}=\phi_{2 m-1,1}\right) \\
& =-\operatorname{coef}\left(h_{2 m}^{+}, \phi_{2 m, 0}\right)+(2 N \kappa+2 m+2) \operatorname{coef}\left(h_{2 m}^{+}, \phi_{2 m, 2}\right) \\
& =-1+(N \kappa+m+1) \frac{g_{1}^{o}(N \kappa-\kappa+m)}{N \kappa+m+1}=N \kappa-\kappa+m .
\end{aligned}
$$

For $\left(T_{1}-T_{2}\right) h_{n}^{+}$, substitute $n=2 m+1, j=1,3$ in Formula (3) to show

$$
\begin{aligned}
& \operatorname{coef}\left(\left(T_{1}-T_{2}\right) h_{2 m+1}^{+}, \psi_{2 m, 1}\right) \\
& =-(2 N \kappa-2 \kappa+2 m+3) \operatorname{coef}\left(h_{2 m+1}^{+}, \phi_{2 m+1,1}\right) \\
& +(2 N \kappa+2 m+4) \operatorname{coef}\left(h_{2 m+1}^{+}, \phi_{2 m+1,3}\right) \\
& =-(2 N \kappa-2 \kappa+2 m+3)+(N \kappa+m+2) \frac{g_{1}^{e}(N \kappa-\kappa+m+1)}{N \kappa+m+2} \\
& =-(N \kappa-\kappa+m) .
\end{aligned}
$$

Next, substitute $n=2 m, j=0,2$ in Formula (3) to show

$$
\begin{aligned}
& \operatorname{coef}\left(\left(T_{1}-T_{2}\right) h_{2 m}^{+}, \psi_{2 m-1,0}\right) \\
& =-(2 N \kappa-2 \kappa+2 m+1) \operatorname{coef}\left(h_{2 m^{\prime}}^{+}, \phi_{2 m, 0}\right)+(2 N \kappa+2 m+2) \operatorname{coef}\left(h_{2 m^{\prime}}^{+}, \phi_{2 m, 2}\right) \\
& =-(2 N \kappa-2 \kappa+2 m+1)+(N \kappa+m+1) \frac{g_{1}^{o}(N \kappa-\kappa+m)}{N \kappa+m+1} \\
& =-(N \kappa-\kappa+m) .
\end{aligned}
$$

This completes the proof of Proposition 2.

To prove Proposition 3, note that the expressions for $u_{1}$ and $u_{2} /(2 \mathrm{i} t)$ have the form

$$
\left(1-2 s t+2 t^{2}\right)^{-2 \kappa-\beta}\left(1-\frac{4\left(1-s^{2}\right) t^{2}}{\left(1-2 s t+2 t^{2}\right)^{2}}\right)^{-\kappa-1},
$$

with $\beta=1$ and 2 , respectively.

Proposition 4. For any $\alpha, \beta$ and $|t|<\frac{1}{\sqrt{2}} \min \left\{\left|s \pm \sqrt{s^{2}-1}\right|\right\}$,

$$
\frac{\left(1-2 s t+2 t^{2}\right)^{2-\beta}}{\left(1-4 s t+8 s^{2} t^{2}-8 s t^{3}+4 t^{4}\right)^{\alpha+1}}=\sum_{n=0}^{\infty} \sum_{j=0}^{\lfloor n / 2\rfloor} S(n, j ; \alpha, \beta) t^{n} s^{n-2 j},
$$

where $S(n, j ; \alpha, \beta)$ is given in Definition 4 .

Proof. Denote the left hand side by $G(s, t ; \alpha, \beta)$. The expansion process begins with

$$
\begin{aligned}
& \left(1-2 s t+2 t^{2}\right)^{-2 \alpha-\beta}\left(1-\frac{4\left(1-s^{2}\right) t^{2}}{\left(1-2 s t+2 t^{2}\right)^{2}}\right)^{-\alpha-1} \\
& =\sum_{\ell=0}^{\infty} \frac{(\alpha+1)}{\ell !} 2^{2 \ell}\left(1-s^{2}\right)^{\ell} t^{2 \ell}\left(1-2 s t+2 t^{2}\right)^{-2 \alpha-\beta-2 \ell} .
\end{aligned}
$$


By a variant of the generating function for Gegenbauer polynomials (see [2] (4.7.23)) with $\lambda>0$,

$$
\begin{aligned}
\left(1-2 s t+2 t^{2}\right)^{-\lambda} & =\left(1+2 t^{2}\right)^{-\lambda} \sum_{k=0}^{\infty} \frac{(\lambda)_{k}}{k !}(2 s t)^{k}\left(1+2 t^{2}\right)^{-k} \\
& =\sum_{k=0}^{\infty} \sum_{m=0}^{\infty} \frac{(\lambda)_{k}}{k !}(2 s t)^{k} \frac{(\lambda+k)_{m}}{m !}\left(-2 t^{2}\right)^{m} \\
& =\sum_{n=0}^{\infty} t^{n} \sum_{m=0}^{\lfloor n / 2\rfloor} \frac{(\lambda)_{n-m}}{(n-2 m) ! m !}(-1)^{m} 2^{n-m} s^{n-2 m},
\end{aligned}
$$

changing the summation index $k=n-2 m$. Combining the expressions results in

$$
\begin{aligned}
& G(s, t ; \alpha, \beta) \\
& =\sum_{\ell=0}^{\infty} \frac{(\alpha+1)_{\ell}}{\ell !}(2 t)^{2 \ell}\left(1-s^{2}\right)^{\ell} \sum_{k=0}^{\infty} t^{k} \sum_{m=0}^{\lfloor k / 2\rfloor} \frac{(2 \alpha+\beta+2 \ell)_{k-m}}{(k-2 m) ! m !}(-1)^{m} 2^{k-m} s^{k-2 m} \\
& =\sum_{n=0}^{\infty} t^{n} \sum_{\ell=0}^{\lfloor n / 2\rfloor} \frac{(\alpha+1)_{\ell}}{\ell !}\left(1-s^{2}\right)^{\ell} \sum_{m=0}^{\lfloor n / 2\rfloor-\ell} \frac{(2 \alpha+\beta+2 \ell)_{n-2 \ell-m}}{(n-2 \ell-2 m) ! m !}(-1)^{m} 2^{n-m} s^{n-2 m-2 \ell,}
\end{aligned}
$$

changing the summation indices to $k=n-2 \ell$. Expand $\left(1-s^{2}\right)^{\ell}=\sum_{i=0}^{\ell}\left(\begin{array}{l}\ell \\ i\end{array}\right)\left(-s^{2}\right)^{\ell-i}$ and change indices replacing $m$ by $j-i$. Then,

$$
\begin{aligned}
& G(s, t ; \alpha, \beta)=\sum_{n=0}^{\infty} t^{n} \sum_{j=0}^{\lfloor n / 2\rfloor} s^{n-2 j} \\
& \times \sum_{\ell=0}^{\lfloor n / 2\rfloor} \sum_{i=\max (0, \ell+j-\lfloor n / 2\rfloor)}^{\min (\ell, j)} \frac{(\alpha+1)_{\ell}(2 \alpha+\beta+2 \ell)_{n-2 \ell-j+i}}{i !(\ell-i) !(j-i) !(n-2 \ell-2 j+2 i) !}(-1)^{\ell+j} 2^{n-j+i} .
\end{aligned}
$$

The summation limits on $i$ are derived from the bounds $0 \leq i \leq \ell, 0 \leq i \leq j$, and $n-2 \ell-$ $2 j+2 i \geq 0$. The last bound implies $i \geq \ell+j-\frac{n}{2}$ (if $n=2 m+1$, the bound is $i \geq \ell+j-m$ and $m=\lfloor n / 2\rfloor)$. The bounds for $s, t$ imply that the two factors $\left(1-2 x s t+x^{2} t^{2}\right)$ for $x=1 \pm \mathrm{i}$ do not vanish for $|\sqrt{2} t|<\min \left|s \pm \sqrt{s^{2}-1}\right|$, and this is sufficient for convergence of the series (if $s=\frac{1}{2}\left(z+z^{-1}\right)$, then the convergence requirement is $|\sqrt{2} t|<\min \left(|z|,|z|^{-1}\right)$ ). This completes the proof for the formula for $S(n, j ; \alpha, \beta)$.

Investigating harmonic polynomials in $p_{1}, p_{2}, p_{3}$ that are $\langle\cdot, \cdot\rangle_{\mathcal{K}}$-orthogonal to the planar polynomials might be a plausible topic for further research.

\section{Conclusions}

There is a well-developed theory of nonsymmetric and symmetric Jack polynomials associated with the symmetric groups. They provide an orthogonal basis for the inner product $\langle f, g\rangle_{\kappa^{\prime}}$, described in Section 3. However these polynomials do not have straightforward formulas for the action of $\Delta_{\kappa}$ and thus the problem of constructing harmonic polynomials appears to require a different approach. The harmonic polynomials would be used to provide an orthogonal basis of polynomials for the Gaussian-type inner product $\langle f, g\rangle_{G}$. The present paper has described the beginnings of a method for this construction.

Conflicts of Interest: The author declares no conflict of interest. 


\section{References}

1. Dunkl, C.; Xu, Y. Orthogonal Polynomials of Several Variables, 2nd ed.; Encyclopedia of Mathematics and Its Applications 155; Cambridge University Press: Cambridge, UK, 2014.

2. Szegö, G. Orthogonal Polynomials, 3rd ed.; American Mathematical Society Colloquium Publications: Providence, RI, USA, 1967; Volume 23.

3. Dunkl, C. Planar harmonic polynomials of type B. J. Phys. A Math. Gen. 1999, 32, 8095-8110.

4. Askey, R. Some basic hypergeometric extensions of integrals of Selberg and Andrews. SIAM J. Math. Anal. 1980, 11, 938-951.

5. Etingof, P. A uniform proof of the Macdonald-Mehta-Opdam identity for finite Coxeter groups. Math. Res. Lett. 2010, 17, 277-284.

6. De Bie, H.; Genest, V.; Vinet, L. A Dirac-Dunkl equation on $S^{2}$ and the Bannai-Ito algebra. Commun. Math. Phys. 2016, 344, 447-464.

7. De Bie, H.; Genest, V.; Vinet, L. The $\mathbb{Z}_{2}^{n}$ Dirac-Dunkl operator and a higher rank Bannai-Ito algebra. Adv. Math. 2015, arXiv:1511.02177.

(C) 2016 by the author; licensee MDPI, Basel, Switzerland. This article is an open access article distributed under the terms and conditions of the Creative Commons Attribution (CC-BY) license (http://creativecommons.org/licenses/by/4.0/). 